\title{
Grasping the second law of thermodynamics at university: The consistency of macroscopic and microscopic explanations
}

\author{
Risto Leinonen," Mervi A. Asikainen, and Pekka E. Hirvonen \\ Department of Physics and Mathematics, University of Eastern Finland, \\ Yliopistokatu 7, 80100 Joensuu, Finland
}

(Received 14 August 2014; published 23 September 2015)

\begin{abstract}
[This paper is part of the Focused Collection on Upper Division Physics Courses.] This study concentrates on evaluating the consistency of upper-division students' use of the second law of thermodynamics at macroscopic and microscopic levels. Data were collected by means of a paper and pencil test $(N=48)$ focusing on the macroscopic and microscopic features of the second law concerned with heat transfer processes. The data analysis was based on a qualitative content analysis where students' responses to the macroscopic- and microscopic-level items were categorized to provide insight into the consistency of the students' ideas; if students relied on the same idea at both levels, they ended up in the same category at both levels, and their use of the second law was consistent. The most essential finding is that a majority of students, $52 \%-69 \%$ depending on the physical system under evaluation, used the second law of thermodynamics consistently at macroscopic and microscopic levels; approximately $40 \%$ of the students used it correctly in terms of physics while others relied on erroneous ideas, such as the idea of conserving entropy. The most common inconsistency harbored by $10 \%-15 \%$ of the students (depending on the physical system under evaluation) was students' tendency to consider the number of accessible microstates to remain constant even if the entropy was stated to increase in a similar process; other inconsistencies were only seen in the answers of a few students. In order to address the observed inconsistencies, we would suggest that lecturers should utilize tasks that challenge students to evaluate phenomena at macroscopic and microscopic levels concurrently and tasks that would guide students in their search for contradictions in their thinking.
\end{abstract}

DOI: 10.1103/PhysRevSTPER.11.020122

PACS numbers: 01.40.Fk, 05.20.-y, 05.70.-a

\section{INTRODUCTION}

\section{A. Entropy and the second law of thermodynamics}

Clausius originally defined the concept of entropy as "the thing that increases by $Q / T$ when heat $Q$ enters a system at temperature $T^{\prime \prime}$ [1]. This idea applies to differential changes as well, and hence, differential change in entropy $S$ can be written $d S=d Q / T$.

When changes in the entropies for two or more objects exchanging energy due to temperature differences are calculated, we notice that the entropy of an object with smaller temperature increases more than the entropy of an object with greater temperature decreases. Hence, the sum of entropy changes is positive, yielding the following result: The net entropy of an isolated system increases in spontaneous processes [2]. The aforementioned statement is referred to as the second law of thermodynamics $[1,2]$.

This statement is useful when predicting the behavior of processes and explaining the functionality of heat engines, for example, but it does not tell what entropy means. In order to describe its characteristics, microscopic models

"risto.leinonen@uef.fi

Published by the American Physical Society under the terms of the Creative Commons Attribution 3.0 License. Further distribution of this work must maintain attribution to the author(s) and the published article's title, journal citation, and DOI. and statistics need to be utilized. Hence, in this article we describe the statistical nature of entropy for solids in a succinct manner [1].

We can describe any solid as consisting of atoms modeled as identical oscillators that can store energy quanta; the same is analogously true for mugs containing balls. When we know the number of oscillators and energy quanta, we can calculate the number of ways in which this energy can be divided among the oscillators. The number of possibilities for achieving this particular stage is called a multiplicity [1].

When two separate solids exchange energy quanta freely, we can estimate the multiplicity of the whole system. We observe that there may be either one or a few states of energy distribution with a significantly greater multiplicity. When the numbers of oscillators and energy quanta are large, $10^{20}$ or more in magnitude, the probability of the most probable state approaches $100 \%$, and fluctuations from that state become negligible. This is one form of the second law of thermodynamics: any system large enough will end up at the most probable, practically inevitable, state [1]. This statement is also true for other types of systems, such as gaseous systems, but the details concerning the second law of thermodynamics for ideal gases is omitted from the article for the sake of brevity.

An interesting finding emerges when the temperatures of two objects exchanging energy freely are calculated; in the most probable state, both objects will have the same 
temperature. This is well in agreement with the previous statement about the macroscopic form of the second law of thermodynamics: the net entropy will increase when energy flows from the higher temperature to the lower temperature. The connection for the multiplicity $\omega$ and entropy $S$ can be specified with the equation $S=k \ln \omega$, where $k$ stands for the Boltzmann constant [1].

When evaluating the largest possible system, the Universe, a consequence of the second law is that the Universe is inevitably proceeding toward a state of the greatest entropy and multiplicity.

\section{B. Previous research}

Referring to entropy as disorder is a typical problem faced by students [3-8]. Admittedly, the idea of the most probable macrostate can be approached using this kind of analogy, but this cannot be considered to be a thorough definition or explanation, due to the ambiguity of the term disorder. Frequently, the term is used with no further explanation of its meaning, or it is referred to from the perspective of visual disorder or chaos, as in the case of a messy room [4-8]. Moreover, the term disorder can be emotionally charged, which is unnecessary when defining concepts in physics [5]. Students' notions regarding disorder have also included references to molecular motion, collisions, and instability $[3,7]$.

Another significant problem involves misunderstanding entropy as a conserved quantity. This conception has been witnessed in the context of heat transfer processes and also of the crystallization of supercooled liquids $[3,7,9]$. This may be related to the problem of confusing entropy with other concepts, such as temperature, energy, heat, enthalpy, or the kinetic energy of particles [4,6-9]. However, it is not always clear if students parallel these concepts or if they cannot specify the difference precisely. For example, a conservation argument might be related to confusing entropy with energy, but not necessarily.

Another problem is "overinterpretation" of the second law of thermodynamics so that entropy is understood as something that cannot be decreased even locally $[3,6,9,10]$. This has been observed in the contexts of the heat transfer processes, ideal gas processes, and some chemical reactions $[6,9,10]$.

Various other problems have also been documented. The other concepts related to the second law, such as a system, surroundings, and the universe, have been shown to be problematic for students to distinguish among [4,6,9,11]. For example, some students tend to claim that the entropy of the Universe is unaffected by spontaneous processes, which indicates that their understanding of the concept of the universe is vague [9]. Thermal equilibrium as a consequence of the second law has been shown to be problematic for students to understand [12]. Some students seem not to realize the relevance of the second law nor the factors affecting entropy. In addition, problems related to understanding the first law of thermodynamics may affect students' understanding of the second law $[7,13]$.

When the literature review is widened to include the connection between macroscopic and microscopic levels in thermal physics topics other than simply the second law of thermodynamics, interesting results can be found. For example, students tend to relate the concept of pressure erroneously to the motion of gas particles or they misunderstand the role of the mass of a particle with regard to pressure. Moreover, students have a tendency to solely concentrate on the average change in momentum and to ignore the incident flux as a factor affecting pressure, or vice versa $[14,15]$.

The concept of temperature is often explained erroneously in terms of microscopic models. Referring to the collisions among the particles as a reason for the increase in temperature is one of the erroneous ideas reported. The other rather prevalent idea is that students assume an incorrect relationship between temperature and particle density $[14,15]$.

Even the ostensibly simple concept of volume is sometimes misunderstood at the microscopic level when evaluating gas systems. Students tend to parallel the number of particles with the volume [14-16]. The other typical problem is to treat the size of a particle as an important factor in the ideal gas model [15].

Some results have been published concerning the underlying ideas related to the concepts of microstate, multiplicity, and probability in the context of mugs containing balls. It was shown that some students have problems in calculating the multiplicities for systems consisting of two entities, and often they rely on adding together the individual multiplicities, rather than multiplying them. Moreover, the task related to calculating probabilities for two simple systems revealed that students may sometimes confuse the concepts of macrostate and microstate [17].

\section{Research question, rationale, and consistency}

The literature review presented above shows that there exists a wealth of evidence concerning the problems that students face with the second law of thermodynamics. Some findings are related to the macroscopic features, while others address the microscopic ones. Nevertheless, we have not discovered any studies that address the consistency of students' ideas concerning the second law at both macroscopic and microscopic levels, even if their connection is essential for understanding the law thoroughly. Hence, the present article addresses this topic by means of the following research question:

How consistent is students' use of the second law of thermodynamics at macroscopic and microscopic levels in the context of heat transfer processes?

The rationale for the study emerges from the importance of the second law of thermodynamics for future scientists, 
teachers, and engineers. Understanding the law at the macroscopic level is a prerequisite for understanding the limitations that the law poses in the case of natural phenomena, processes, and technological innovations. In addition, the microscopic interpretation of the law addresses the question "why?," which is an essential question for scientists and teachers. Moreover, grasping the connection between these two interpretations is essential for students because it is probably the first, if not the sole, occasion on which they can see how a generalizable principle of physics is based on microscopic models and statistical physics.

The concept of consistency is a familiar term in everyday language. However, there is some variation in the use of the concept of consistency in education research [18-22], as explicitly noted by Tongchai et al. [18]. Typically, the use of consistency has been rather pragmatic in such studies, and the theoretical framework underlying the concept has received only minor attention. In what follows, we will attempt to explain the meaning of consistency as it is used in the course of this article.

One description of consistency is a demand for the absence of internal contradictions. This means that a consistent explanation should not provide two opposing predictions, for example [23]. This is thoroughly in agreement with our use of the concept because we concentrate on evaluating possible contradictions in the responses of individual students. This description is seen as the most important description of consistency in this study, and it is also the most obvious similarity between the use of the concept in this study and in other studies [18-22].

Tongchai et al. [18] identify two interpretations concerning the different types of consistency: it can be evaluated either at the level of the individual student or at the level of the class. In this study, the consistency of individual student's ideas has been subjected to evaluation, as in numerous other studies $[18,19,21]$. This means that instead of evaluating and comparing the percentages of different responses or ideas within cohorts, we categorize single students' responses individually so that we can see if a particular student is using their ideas consistently.

In this study, the internal consistency of the answers provided by individual students will, therefore, be evaluated. This means that our main focus is not on evaluating to see if students can deliver correct responses in terms of physics but rather on discovering if their answers are logical in relation to each other. ${ }^{1}$ This means that correctness is not a precondition for consistency; a person can possess consistent ideas even if they are erroneous in terms of physics and cannot provide correct predictions for particular phenomena. For example, it has been reported

\footnotetext{
${ }^{1}$ In other words, the answers of a student are considered to be consistent if they could be right if "the Universe worked differently."
}

that students' ideas about motion can be internally consistent even if they lack physical correctness [24].

Typically, when evaluating consistency, similar themes may be addressed in different contexts. This typically refers to modifying the physical situation under evaluation [18-21]. For example, students' ideas about basic mechanics have been studied in the contexts of different types of motion [21]. In the present study, the context remains similar and the phenomenon under evaluation, heat transfer, remains the same. The difference emerges from the evaluation of students' use of the second law of thermodynamics on two levels, namely, the macroscopic and microscopic levels. This is a novel approach to the study of consistency. Because a profound understanding of the second law of thermodynamics requires that it be understood at both a macroscopic and a microscopic level, this type of consistency needs to be evaluated.

To summarize our description of consistency, the concept refers to how systematically students are able to use their ideas when the level (macroscopic level versus microscopic level) of evaluating the phenomenon is modified. Because of the similar contexts and assignments that are described later in this article, the most clearly dominant variable emerges from the level of the evaluation of the phenomenon. The answers provided do not have to be physically correct to be consistent, and we are interested in evaluating the internal consistency at an individual student level.

With respect to the previous studies, consistency in students' responses and explanations has been studied in various areas of physics, such as motion, forces, mechanical waves, combustion, and density and pressure [18-22]. Research has also been conducted in the consistency of students' interpretation of multiple representations, teaching approach and student achievement, and epistemological issues [25-27]. What seems to be common to most of the studies is that students tend to use their physics ideas inconsistently [18-22]. For example, it has been shown that in the case of mechanics, both correct ideas and erroneous ideas, such as "motion implies force," are used inconsistently, even in similar contexts [21]. Another example emerges from using the concept of temperature: using it seems to be dependent on the context when students' concept descriptions are inconsistent with their use of the concept when explaining a physical phenomenon [28]. Students' ideas about mechanical waves are also shown to be inconsistent and with a large number of variations, depending on their previous studies and maturity [18]. These are individual examples, but they show that inconsistency can dominate greatly in students' thinking.

\section{METHODS}

This section introduces the context of this research project. Moreover, the methodological choices concerning the collection and analysis of the data are introduced. 


\section{A. Context}

The collection of the data took place in the upperdivision course in thermal physics at the Department of Physics and Mathematics at the University of Eastern Finland. The course is a compulsory course within the entity of intermediate physics studies and it is designed for second-year physics majors and minors.

The course has approximately 50 participants annually, all of them majoring in mathematics, physics, chemistry, or computer science. Teachers and scientists are educated in the course. More than half of the students have taken a course in thermal physics at upper secondary school. At the university level, the students have taken an introductorylevel entity in basic physics (4 lecture-based courses and one laboratory course). During the Basic Physics II course, the basics of thermal physics have been covered, but the second law of thermodynamics is omitted from the course content. In addition, students have studied kinematics, mechanics, waves, oscillations, electricity, and magnetism. Students majoring in chemistry have studied $1-3$ courses of physical chemistry at university. However, those with different study backgrounds are not distinguished in this article because it was revealed during the analysis that the differences among the subgroups were insignificant and not generalizable, and the subgroups would be rather small.

The course is based on the textbook by Schroeder. Its main emphases are on the following topics: the ideal gas law and model, equipartition of energy, the first law of thermodynamics, transfer processes, the second law of thermodynamics statistically, the second law of thermodynamics classically, and heat engines and refrigerators [1]. Students have access to PowerPoint slides that form the framework for lectures, and only a few students use the textbook during the course. The lecture material is supplemented with numerous examples and explanations during the lectures, so the PowerPoint slides by themselves are unlikely to be sufficient for students to grasp the course content.

Teaching is implemented with the aid of lectures and homework sessions. During the lectures ( 4 class periods per week, $45 \mathrm{~min}$ each), a lecturer goes through the topics selected from the book and presents examples concerning the current topic. Students are encouraged to ask questions during the lectures, and occasionally the lectures are supplemented by activating elements, such as pair discussions. Generally speaking, a course has 30 class periods of lectures.

During homework sessions (2 class periods per week) the students present the solutions that they have produced in their homework to their fellow students. The homework sessions are scheduled to follow one week behind the lectures so that the themes covered in the homework sessions will be familiar in light of the preceding lectures. A teaching assistant comments, supplements, and corrects student responses when required, and occasionally shows example solutions to the tasks. Students are rewarded with extra points for the homework done in the course evaluation (maximum 10\% of the points of the course exam). Generally speaking, a course has 14 class periods of homework sessions.

Participation in the lectures and homework sessions is voluntary for students. Typically, the percentage of the students participating in lectures and homework sessions is approximately $50 \%$. The course grade is determined by the points received in the course exam held at the end of the course, supplemented by possible extra points received in the homework sessions.

\section{B. Data collection methods}

Our present data were collected in two consecutive years during the current decade. ${ }^{2}$ Teachers, content, and the students' preceding studies remained the same in these years and hence the samples are not distinguished in the analysis. The data collection was conducted by means of paper and pencil tests.

We wanted to test students' understanding of the second law and entropy at the macroscopic and microscopic levels. The paper and pencil test used in the data collection was constructed based on the tasks developed by Christensen et al. [9]; these items (1a-1d in our test) test students' understanding of the macroscopic characteristics of entropy and the second law. They were also used as a starting point for designing items addressing the same themes at the microscopic level (2a-2d in our test). Basically, we developed a "microscopic counterpart" for each of the items in the original test [9]. The context was modified slightly so that the connection would not be too selfevident for students, but the physical phenomenon under evaluation was the same in both sets of items, namely, heat transfer. The test as a whole can be found in the Appendix, accompanied by the required responses and explanations.

Our students were given the paper and pencil test, consisting of the aforementioned items, once the content necessary to succeed in the test had been presented in the lectures and homework sessions. Thus, both the macroscopic and microscopic interpretations related to the second law and related concepts had been introduced in lectures (some 12 class periods) and homework sessions (some 4 class periods). The two sets of test items were taken at the same time by the students. Data collection took place at the end of each class, and the students were allowed to use as much as time as they required and to leave the lecture hall once they had handed in their response papers. In general, the students needed $10-15$ min to take the test.

\footnotetext{
${ }^{2}$ The exact years are omitted to protect the anonymity of the research subjects.
} 


\section{Analyzing data}

Our test questions enabled us to place students' responses in certain predetermined categories (see the Appendix) concerning changes in entropy or in the number of accessible microstates. This categorization offered a base for analyzing students' ideas further based on the explanations that the students used to construct their responses. Analysis of the explanations was based on a qualitative content analysis [29,30].

When ideas are discussed in the course of this article, we refer to the student's understanding of the physical situation under evaluation that appears to underlie their answers. These ideas can be correct or erroneous in terms of physics. Because the phenomenon under evaluation, namely, heat transfer, can be expected to be explained at both the macroscopic and microscopic levels, these ideas cannot be identical at both levels, even if they are interpreted as the same. For example, the idea of increasing entropy in spontaneous processes in item $1 \mathrm{c}$ is claimed to be the same as the idea of the system consisting of two objects ending up in a state with the greatest number of accessible microstates in item 2c. Similarly, the idea of the conservation of entropy and the idea of the number of accessible microstates remaining constant are interpreted as the same idea.

First, students' responses to the two sets of test items used were analyzed individually in order to see the ideas students had in terms of their understanding of the second law at macroscopic and microscopic levels in heat transfer processes. In order to support this analysis, we analyzed the consistency across the subparts of both individual set of items (1a-1d and 2a-2d).

Next, these two data sets were compared student by student to see if their use of the second law was consistent at macroscopic and microscopic levels. This aspect was based on the students' ideas that were observed when the data sets were analyzed individually; in turn, these findings guided our formulation of the categories that could be identified as common to the explanations at both the macroscopic and microscopic levels. This procedure included reducing and combining categories. In practice, formulation of the categories was a cyclic process that frequently included the specification and combination of categories and recategorization of the responses. By this means, we were able to see whether some of the students possibly understood the law at one level but not at the other, or whether students had differing problems when applying the law at these two levels.

\section{RESULTS}

This section presents the results that we obtained. First, we present an overview of students' performance related to the items addressing the macroscopic features of the second law of thermodynamics. Our second focus is then on students' ideas concerning the microscopic features of the second law of thermodynamics. Third, we evaluate the consistency of
TABLE I. Percentages of students' categorized responses related to the items addressing the macroscopic nature of the second law and changes in entropy in a heat transfer process. The percentage of correct responses is featured in boldface $N=48$.

\begin{tabular}{lcccc}
\hline \hline & \multicolumn{4}{c}{ Entity under inspection } \\
\cline { 2 - 5 } & $1 \mathrm{a}$. & $1 \mathrm{~b}$. & $1 \mathrm{c}$. & $1 \mathrm{~d}$. \\
Entropy & Object & Air & Object + air & Universe \\
\hline Increases & $8 \%$ & $10 \%$ & $\mathbf{6 3} \%$ & $\mathbf{6 9} \%$ \\
Decreases & $8 \%$ & $2 \%$ & $0 \%$ & $0 \%$ \\
Remains the same & $0 \%$ & $2 \%$ & $29 \%$ & $19 \%$ \\
Change in entropy & $\mathbf{7 9 \%}$ & $\mathbf{7 9} \%$ & $4 \%$ & $8 \%$ \\
$\quad$ cannot be determined & & & & \\
Empty & $4 \%$ & $6 \%$ & $4 \%$ & $4 \%$ \\
\hline \hline
\end{tabular}

students' ideas about the second law of thermodynamics at both macroscopic and microscopic levels.

\section{A. Entropy and the second law, macroscopically}

Students' ideas about the macroscopic features of the second law of thermodynamics were evaluated with the aid of the test questions 1a-1d (see the Appendix) [9]. Students' responses to each of these questions were placed in five categories, as seen in Table I. The categories emerge from the predetermined choices in the test items.

The categorization shown in Table I pays no attention to the explanations underlying students' conclusions. In the following sections, we will shed light on these.

\section{Consistency among macroscopic items}

In order to support the next stage of our analysis, we evaluated the consistency among the students' answers in items 1a-1d. This was done by analyzing the logic connecting the answers. The criteria for consistency followed those described previously in this article, the most important characteristic being the absence of internal contradictions.

To illustrate our interpretation of consistency, we present sample quotes from students who gave consistent and correct, consistent and erroneous, and inconsistent ${ }^{3}$ answers in items $1 \mathrm{a}-1 \mathrm{~d}$ in Table II. The reason for distinguishing between the two apparent types of consistent sets of answers, the correct and the erroneous ones, is that we wish to illustrate the types of answers that the students provided. Regarding the category of consistent and erroneous answers, any flaw in terms of the actual physics means that the set of responses would be placed in this category, even if some of the answers appeared to be correct.

\footnotetext{
${ }^{3}$ Inconsistent answers are not distinguished to the two logical groups (inconsistent + inaccurate, inconsistent + partially accurate) due to our data; there were not representative examples for both of these groups.
} 
TABLE II. Sample quotes of the different types of responses in terms of their consistency within the four items (1a-1d) concerned with changes in macroscopic entropy. Two types (correct and erroneous) of consistent sets of answers and one set of inconsistent answers are provided. The inconsistency is in boldface in the last example.

\begin{tabular}{|c|c|c|c|c|}
\hline \multirow[b]{2}{*}{ Type of consistency } & \multicolumn{4}{|c|}{ Change in entropy for the... } \\
\hline & 1a. Object & 1b. Air & 1c. Object + air & 1d. Universe \\
\hline $\begin{array}{l}\text { 1. Consistent } \\
\text { and correct }\end{array}$ & $\begin{array}{l}\text { It cannot be determined } \\
\text { because we do not } \\
\text { know if the object is } \\
\text { warmer than the air } \\
\text { or not }\end{array}$ & $\begin{array}{l}\text { It cannot be determined } \\
\text { because we do not } \\
\text { know if the air is } \\
\text { warmer than the object } \\
\text { or not }\end{array}$ & $\begin{array}{l}\text { It increases because the } \\
\text { decrease in entropy in } \\
\text { another entity < } \\
\text { increase in entropy in } \\
\text { another entity }\end{array}$ & $\begin{array}{l}\text { It increases because the } \\
\text { entropy in the room } \\
\text { has already increased }\end{array}$ \\
\hline $\begin{array}{l}\text { 2. Consistent and } \\
\text { erroneous }\end{array}$ & $\begin{array}{l}\text { It cannot be } \\
\text { determined. The } \\
\text { entropy has changed } \\
\text { because energy can } \\
\text { be transferred, and } \\
\text { the object and the air } \\
\text { have different } \\
\text { temperatures; but it } \\
\text { cannot be determined } \\
\text { whether the entropy } \\
\text { of the object has } \\
\text { increased or } \\
\text { decreased when it is } \\
\text { unknown if the } \\
\text { object is hotter or } \\
\text { colder than the air }\end{array}$ & $\begin{array}{l}\text { It cannot be determined. } \\
\text { We do now know } \\
\text { whether the } \\
\text { temperature is higher } \\
\text { for the object or for } \\
\text { the air }\end{array}$ & $\begin{array}{l}\text { It remains the same: } \\
\text { There is no energy } \\
\text { exchange between the } \\
\text { other world/the walls } \\
\rightarrow \text { No energy } \\
\text { exchange between the } \\
\text { outside world }\end{array}$ & $\begin{array}{l}\text { It remains the same. The } \\
\text { summed entropy did } \\
\text { not increase } \rightarrow \text { The } \\
\text { entropy of the } \\
\text { Universe did not } \\
\text { increase }\end{array}$ \\
\hline 3. Inconsistent & $\begin{array}{l}\text { There is an energy } \\
\text { exchange between } \\
\text { the object and the air, } \\
\text { so the entropy has } \\
\text { changed. Because } \\
\text { temperatures are not } \\
\text { given, it cannot be } \\
\text { determined whether } \\
\text { it will increase or } \\
\text { decrease }\end{array}$ & $\begin{array}{l}\text { It changes to some extent } \\
\text { because there is some } \\
\text { energy exchange } \\
\text { between the object and } \\
\text { the air, since they have } \\
\text { different temperatures. } \\
\text { However, the change } \\
\text { is not significant }\end{array}$ & $\begin{array}{l}\text { It remains the same } \\
\text { when the entropy has } \\
\text { increased in one and } \\
\text { decreased in the } \\
\text { other. So, the sum of } \\
\text { the entropies is zero }\end{array}$ & $\begin{array}{l}\text { The entropy in the } \\
\text { Universe increases } \\
\text { continuously. A new } \\
\text { entropy is created } \\
\text { during the process, } \\
\text { and the entropy } \\
\text { existing outside the } \\
\text { system remains } \\
\text { unchanged }\end{array}$ \\
\hline
\end{tabular}

Analyzing the number of inconsistent responses in a particular type, as seen in Table II, revealed that a large majority of the students had provided consistent answers to items 1a-1d; only three of the 48 students provided answers that were considered to be inconsistent. In addition to the example in Table II, two other students provided answers with inconsistency in items $1 \mathrm{c}$ and $1 \mathrm{~d}$. However, these two students seem to confuse the concepts of "surroundings" and "the universe," so this problem appears to be related to these concepts, and it is difficult to conclude anything about their ideas concerning the changes in entropy. The small number of students with internal inconsistencies at a macroscopic level is a supporting result for our analysis, as may be seen in the following sections.

\section{Changes in entropies for the object and the air in the room}

$79 \%$ of the students drew the correct conclusion in items $1 \mathrm{a}$ and $1 \mathrm{~b}$ (see Table I) addressing entropy changes for individual entities. However, the correct conclusion (a change in entropy cannot be determined) can be reached by means of various, also erroneous, explanations. Thus, we would refer to our previous statement about the internal consistency of students' answers at macroscopic items $1 \mathrm{a}-1 \mathrm{~d}$ to enhance the reliability ${ }^{4}$ of our analysis. This means that if the explanation provided by a student in response to item $1 \mathrm{a}$ or $1 \mathrm{~b}$ was difficult or impossible to interpret, we checked the student's answer in item 1c in order to get confirmation for their ideas. This is a justified solution based on the high level of consistency seen in items $1 \mathrm{a}-1 \mathrm{c}$.

\footnotetext{
${ }^{4}$ The quotes seen in Table II illustrate how students' answers should not necessarily be interpreted as individual entities because students' answers in items $1 \mathrm{a}$ and $1 \mathrm{~b}$ might appear to be correct even if their answer to item 1c shows evident flaws in terms of physics.
} 
For example, the set of answers in the second category in Table II shows that even if the answers in items $1 \mathrm{a}$ and $1 \mathrm{~b}$ appear to be correct, an erroneous idea about the conservation of entropy seems to underlie their formulation, as can be seen in the case of the answer to item 1c. Thus, this type of answer can be stated to treat entropy as a conserved quantity. The explanations that could not be specified further are omitted from the text for the sake of brevity.

The following types of explanations were observed among the students who had drawn the correct conclusion (79\% for both items; see Table I) in these items (see additional original quotes from the students' explanations in Supplemental Material [31]):

- Correct understanding of entropy (1a, 54\%; 1b, 54\%)

- Entropy is seen as a conserved quantity (1a, 25\%; $1 \mathrm{~b}, 23 \%)$

- The meaning of entropy remains vague (1a, 0\%; $1 b, 2 \%)$

In the categories "Entropy increases" (1a, $8 \% ; 1 \mathrm{~b}, 10 \%$; see Table I) and "Entropy decreases" (1a, $8 \% ; 1$ b, 2\%), the following types of explanations were observed:

- An assumption that local entropy always increases (1a, 4\%; 1b, 4\%)

- Unnecessary assumptions about the temperature difference between the entities (1a, 12\%; 1b, 6\%)

The explanation for the response stating that the entropy of the air in the room would remain unchanged during the process paralleled the change in entropy with the change in temperature (1a, $0 \% ; 1 \mathrm{~b}, 2 \%)$.

\section{Changes in entropy for the combined system of the object and the air in the room, and for the Universe}

$63 \%$ of the students drew the correct conclusion for the entropy changes in items 1c (object + air) and 69\% 1d (the Universe); see Table I. These students used the following types of explanations in the aforementioned items. The explanations that could not be specified further are omitted from the text for the sake of brevity.

- Correct desired explanations (1c, 56\%; 1d, 56\%)

- References to the expansion of the Universe (1c, $0 \%$; $1 \mathrm{~d}, 6 \%)$

- References to disorder (1c, 0\%; 1d, 2\%)

- References to the tendency of temperatures to even out (1c, $0 \% ; 1 \mathrm{~d}, 2 \%)$

Students' responses stating that the entropy would remain unchanged (see Table I) in items 1c (29\%) and 1d (19\%) suggested that they regarded entropy as a conserved quantity. The explanations for these responses assumed the following main themes:

- Zero energy change between the system and its surroundings (1c, 13\%; 1d, 13\%)

- The entropies leaving and entering the entities are equal (1c, $8 \% ; 1 \mathrm{~d}, 0 \%)$
- The massive size of the Universe as a reason (1c, 0\%; $1 \mathrm{~d}, 2 \%)$

A few responses (1c, 4\%; 1d, 8\%; see Table I) in the category "Cannot be determined" followed the aforementioned themes, and hence they are not discussed here.

\section{Summary}

When evaluating percentages of the correct responses seen in Table I, students seemed to understand the second law of thermodynamics and the macroscopic nature of entropy rather well. However, it was shown that there was also a tendency to understand entropy as a conserved quantity. A few students (4\%) seemed to think that entropy could not be decreased even locally.

\section{B. Second law microscopically}

Students' ideas about the microscopic aspects of the second law of thermodynamics were evaluated with the aid of test items 2a-2d (see the Appendix). As with task 1, the students' responses were placed in predetermined (the number of accessible microstates increases, decreases, or remains the same, and empty) categories. This categorization can be seen in Table III. Compared to task 1, the absence of the "cannot be determined" category is due to a difference in the assignment; the temperatures of the objects could be inferred and hence it was not offered as an option.

The categorization shown in Table III pays no attention to the explanations that students used in defining their responses, and these are discussed in the following sections.

\section{Consistency among microscopic items}

As with items 1a-1d, the consistency between the answers in microscopic items $2 \mathrm{a}-2 \mathrm{~d}$ was evaluated in order to support further analysis. The procedure was identical to that described in Sec III A 1. The reason for this approach was to discover whether the students had been able to provide consistent answers for one set of items. Sample

TABLE III. Percentages of students' categorized responses in the items addressing the microscopic nature of entropy and the second law of thermodynamics. The percentage of correct responses is shown in boldface $N=48$.

\begin{tabular}{|c|c|c|c|c|}
\hline \multirow{2}{*}{$\begin{array}{l}\text { Number of } \\
\text { accessible } \\
\text { microstates }\end{array}$} & \multicolumn{4}{|c|}{ Entity } \\
\hline & $\begin{array}{c}2 \mathrm{a} . \\
\text { Object A }\end{array}$ & $\begin{array}{c}2 \mathrm{~b} . \\
\text { Object B }\end{array}$ & $\begin{array}{c}\text { 2c. } \\
\text { Objects } A+B\end{array}$ & $\begin{array}{c}2 \mathrm{~d} . \\
\text { Universe }\end{array}$ \\
\hline Increases & $4 \%$ & $90 \%$ & $44 \%$ & $44 \%$ \\
\hline Decreases & $\mathbf{8 5} \%$ & $0 \%$ & $0 \%$ & $2 \%$ \\
\hline $\begin{array}{l}\text { Remains } \\
\text { the same }\end{array}$ & $4 \%$ & $4 \%$ & $48 \%$ & $35 \%$ \\
\hline Empty & $6 \%$ & $6 \%$ & $8 \%$ & $19 \%$ \\
\hline
\end{tabular}


TABLE IV. Sample quotes from different types of answers in terms of consistency in items 2a-2d, which were concerned with changes in the number of accessible microstates. Two types (correct and erroneous) of consistent sets of answers and one set of inconsistent answers are shown. The inconsistency is in boldface in the final sample.

\begin{tabular}{|c|c|c|c|c|}
\hline \multirow[b]{2}{*}{ Type of consistency } & \multicolumn{4}{|c|}{ Change in the number of accessible microstates for the... } \\
\hline & 1a. Object A & 1b. Object B & 1c. Objects $A+B$ & 1d. Universe \\
\hline 1. Consistent and correct & $\begin{array}{l}\text { It decreases because the } \\
\text { number of energy } \\
\text { quanta declines, so the } \\
\text { number of their } \\
\text { combinations also } \\
\text { declines }\end{array}$ & $\begin{array}{l}\text { It increases because the } \\
\text { number of energy } \\
\text { quanta is increased, so } \\
\text { the number of their } \\
\text { combinations also } \\
\text { increases }\end{array}$ & $\begin{array}{l}\text { It increases. The number } \\
\text { of microstates achieves } \\
\text { its maximum value in } \\
\text { equilibrium; when the } \\
\text { energy is divided more } \\
\text { evenly, there are more } \\
\text { microstates }\end{array}$ & $\begin{array}{l}\text { It increases because the } \\
\text { entropy is additive }\end{array}$ \\
\hline $\begin{array}{l}\text { 2. Consistent and } \\
\text { erroneous }\end{array}$ & $\begin{array}{l}\text { It decreases because } \\
\text { thermal energy is } \\
\text { transferred to B }\end{array}$ & $\begin{array}{l}\text { It increases because it } \\
\text { receives thermal } \\
\text { energy }\end{array}$ & $\begin{array}{l}\text { It remains the same; there } \\
\text { is no energy or } \\
\text { anything else coming } \\
\text { from anywhere }\end{array}$ & $\begin{array}{l}\text { It remains the same; they } \\
\text { do not "sprout" from } \\
\text { nowhere }\end{array}$ \\
\hline 3. Inconsistent & It decreases & It increases & It increases & $\begin{array}{l}\text { An insulated system } \\
\rightarrow \text { No effect on the } \\
\text { Universe }\end{array}$ \\
\hline
\end{tabular}

quotes from the students' responses with different types of consistencies are shown in Table IV.

Analyzing the numbers of students providing responses with inconsistencies revealed that the students were rather consistent when performing microscopic-level tasks; only four of the 48 students seemed to have inconsistencies in their responses. Three of them used an explanation of a type seen in the last row in Table IV. These answers indicate that the inconsistency was due to a misunderstanding of the meaning of the universe. The one remaining student was inconsistent solely in item $2 \mathrm{~d}$ while he stated that since the number of microstates decreases, it will achieve the maximum value. Based on these findings, we can conclude that the students were rather consistent in their responses to items $2 \mathrm{a}-2 \mathrm{~d}$.

\section{Changes in the numbers of accessible microstates for the two objects exchanging energy}

As seen in Table III, a large majority of the students came to the correct conclusion in items $2 \mathrm{a}(85 \%)$ and $2 \mathrm{~b}(90 \%)$. However, this conclusion may also be achieved with the aid of erroneous explanations. With these types of answers, we made use of our previous finding about the high consistency among items $2 \mathrm{a}-2 \mathrm{~d}$; this was a process that was identical to the one explained in Sec. III A 2. The explanations used by the students drawing the correct conclusions followed the themes presented below [31]. The explanations that could not be specified further are omitted from the text for the sake of brevity.

- Students possess a correct idea of the number of microstates (2a, 40\%; 2b, 40\%)

- The number of accessible microstates is considered to be a conserving quantity $(2 \mathrm{a}, 46 \% ; 2 \mathrm{~b}, 46 \%)$
- An explanation that assumes that the number of accessible microstates always increases (2a, 0\%; $2 \mathrm{~b}, 4 \%$

The percentages of students drawing any erroneous conclusions (the empty answers are omitted from these percentages) remained low $(2 \mathrm{a}, 8 \% ; 2 \mathrm{~b}, 4 \%)$ in these particular tasks, as seen in Table III. One kind of explanation was observed in these responses, and it is a familiar one from the previous listing:

- An explanation assuming that the number of accessible microstates always increases $(2 \mathrm{a}, 4 \% ; 2 \mathrm{~b}, 0 \%)$

Other types of explanations were not observed in these items.

\section{Changes in the number of accessible microstates for the combined system of the two objects, and for the Universe}

The percentages of students providing correct responses to items $2 \mathrm{c}$ and $2 \mathrm{~d}$ were significantly lower than for other items, $44 \%$ in both cases (see Table III). The explanations provided by these students followed the themes shown below [31]. The explanations that could not be specified further are omitted from the text for the sake of brevity:

- Desired explanations based on energy distribution, entropy additivity among the objects, the connection between entropy and the number of microstates, or relying on the idea of two objects as a part of the Universe (2c, 40\%; 2d, 37\%)

- References to a tendency of temperature differences to even out or to the expansion of the Universe (2c, $0 \%$; $2 \mathrm{~d}, 6 \%)$

Numerous students (2c, 48\%; 2d, 35\%; see Table III) concluded that the number of accessible microstates would 
remain unchanged during the process. The following themes were observed in students' explanations [31]:

- Using a compensation argument in a way that students assumed changes in the numbers of microstates of objects A and B to correspond to each other (2c, 13\%; 2d, $0 \%$ )

- The objects were not regarded as a part of the Universe (2c, 6\%; 2d, 6\%)

- Explanations referring to the connection between the number of accessible microstates and entropy (2c, 6\%; $2 \mathrm{~d}, 6 \%$ )

$23 \%$ of students did not provide any explanations for their response in this category.

\section{Summary}

With regard to students' ideas concerning the second law of thermodynamics from the microscopic point of view, we can see similarities with those that we observed previously vis-à-vis the items addressing these themes at the macroscopic level. For example, the number of accessible microstates was considered to be a conserving quantity, just as entropy was. Regarding the connection between entropy and the number of microstates, some students knew the connection but their inadequate understanding of the actual second law per se made them give erroneous explanations.

\section{Consistency}

In this section we show how consistently students used the second law at the macroscopic and microscopic levels. This will be achieved by categorizing students' responses to the matched items and by providing a comparison of the findings.

Our categorization began by finding the most common students' ideas observed with the data sets introduced in the previous sections. The responses to the matched macroscopic and microscopic items provided by individual students were then placed in these categories. If a student's responses followed the same ideas at both microscopic and macroscopic levels, they ended up in the same category at both levels, which signals that the student's ideas were consistent.

\section{Consistency of students' explanations concerning changes in entropy and the number of accessible microstates for individual entities}

Changes in entropies for individual entities were addressed in items $1 \mathrm{a}$ and $1 \mathrm{~b}$, while changes in the numbers of accessible microstates were addressed in items $2 \mathrm{a}$ and $2 \mathrm{~b}$. Thus, when evaluating the consistency of students' ideas at these two levels, we could formulate four item pairs $(1 \mathrm{a}+2 \mathrm{a}, 1 \mathrm{a}+2 \mathrm{~b}, 1 \mathrm{~b}+2 \mathrm{a}$, and $1 \mathrm{~b}+2 \mathrm{~b})$. This is not appropriate if we consider the only minor differences between the responses that students provided to these items; only a few students were categorized differently
TABLE V. Cross tabulation of students' explanations concerning changes in entropy and in the number of accessible microstates for individual objects (items $1 \mathrm{a}$ and $2 \mathrm{a}$ ). $N=48$. Categories 1-4 are the same in columns and rows but for macroscopic (columns) and microscopic (rows) levels.

\begin{tabular}{|c|c|c|c|c|}
\hline \multirow[b]{2}{*}{ Students' explanations } & \multicolumn{4}{|c|}{ Macroscopic level } \\
\hline & 1 & 2 & 3 & 4 \\
\hline \multicolumn{5}{|c|}{ Microscopic level } \\
\hline 1. Correct ideas & $38 \%$ & $0 \%$ & $2 \%$ & $4 \%$ \\
\hline $\begin{array}{l}\text { 2. Entropy is seen as a } \\
\text { conserved quantity }\end{array}$ & $13 \%$ & $25 \%$ & $0 \%$ & $8 \%$ \\
\hline 3. Entropy always increases & $0 \%$ & $0 \%$ & $2 \%$ & $0 \%$ \\
\hline 4. Uncategorized and empty & $4 \%$ & $0 \%$ & $2 \%$ & $2 \%$ \\
\hline
\end{tabular}

in items $1 \mathrm{a}$ and $1 \mathrm{~b}$ or in items $2 \mathrm{a}$ and $2 \mathrm{~b}$. Hence, we decided to concentrate on the combination of tasks 1a and 2a. The cross tabulation of students' explanations in items 1a and 2a can be seen in Table V.

Categories seen in Table $\mathrm{V}$ were the typical ideas ${ }^{5}$ used by the students when explaining the aforementioned items shown in Secs. III A 2 and III B 2. The criteria for the categorization were the following (see Supplemental Material [31] for examples of explanations belonging in each of these categories [31]):

(1) These explanations are correct in terms of physics.

(2) In the case of these explanations, entropy or the number of accessible microstates can be seen to be a conserved quantity. Some of these responses signal some confusion between entropy and energy or between the number of accessible microstates and the number of energy quanta.

(3) These explanations rely on erroneous idea that the entropy or the number of accessible microstates always increases locally.

(4) These students gave either empty responses or responses that could not be placed in these categories. These answers are omitted from the following discussion for the sake of brevity.

The first observation that can be drawn from Table $\mathrm{V}$ is that $38 \%$ of the students are placed in the intersection of categories 1 and 1 . This suggests that these students have consistent and correct ideas about changes in entropy and the number of accessible microstates. With regard to category $2,25 \%$ of the students relied on the same type of explanation at both macroscopic and microscopic levels, which indicates a consistent yet erroneous notion concerning the conservation of entropy. One student consistently used the idea of entropy as something that always increases at both macroscopic and microscopic levels. To sum up the

\footnotetext{
${ }^{5}$ Ideas that are not applied in both items (e.g., drawing assumptions about temperature difference in item 1a when temperatures can be inferred in item 2a) are not shown in this categorization.
} 
TABLE VI. Cross tabulation of students' explanations regarding changes in the entropy and in the number of accessible microstates for the combined systems of two entities undergoing the heat transfer process (items $1 \mathrm{c}$ and $2 \mathrm{c}$ ). $N=48$. Categories 1-3 are the same in columns and rows but for macroscopic (columns) and microscopic (rows) levels.

\begin{tabular}{|c|c|c|c|}
\hline \multirow[b]{2}{*}{ Students' explanations } & \multicolumn{3}{|c|}{ Macroscopic level } \\
\hline & 1 & 2 & 3 \\
\hline \multicolumn{4}{|c|}{ Microscopic level } \\
\hline 1. Correct & $42 \%$ & $2 \%$ & $0 \%$ \\
\hline $\begin{array}{l}\text { 2. Entropy or the number } \\
\text { of accessible microstates } \\
\text { are regarded as a } \\
\text { conserved quantity }\end{array}$ & $15 \%$ & $27 \%$ & $6 \%$ \\
\hline 3. Uncategorized and empty & $4 \%$ & $0 \%$ & $4 \%$ \\
\hline
\end{tabular}

aforementioned results, $65 \%$ of the students were able to provide consistent answers in these items.

Inconsistent answers were witnessed mostly in the intersection of categories 1 and 2: 13\% of the students used entropy correctly but considered the number of accessible microstates to be a conserving quantity. Other inconsistencies were observed only from one student.

\section{Consistency of students' responses concerning changes in entropy and the number of accessible microstates for the combined system of two entities}

Table VI shows students' categorized responses in items $1 \mathrm{c}$ and $2 \mathrm{c}$, which addressed changes in the entropy and in the number of accessible microstates for the combined system of two entities.

The categories seen in Table VI are typical of the ideas used by the students to explain the aforementioned items, as introduced in Secs. III A 3 and III B 3. The criteria used for the categorization are as follows:

(1) This category includes responses stating that the entropy or the number of accessible microstates of the combined system of two entities would increase during the process. Correct responses without explanations were placed in this category. ${ }^{6}$

(2) This category includes responses with various types of explanations concluding that the entropy or the number of accessible microstates of the combined system of two entities would remain unchanged during the process.

\footnotetext{
${ }^{6}$ The reason to place correct responses without any explanations in this category was the fact that it is unlikely to provide correct answers with erroneous explanations in this type of item. Moreover, the number of students giving correct answers without any further explanations in items $1 \mathrm{c}$ and $2 \mathrm{c}$ was rather modest for these items: 3 students and 1 student, respectively.
}

(3) These students gave either empty responses or responses that could not be placed in these categories.

$42 \%$ of the students have been placed in the intersection of categories 1 and 1, indicating that these students could utilize the second law of thermodynamics consistently and correctly. $27 \%$ of the students are seen in the intersection of categories 2 and 2. These students provided consistent, yet erroneous, ideas about entropy or the number of accessible microstates as something that is conserved. The aforementioned percentages imply that $69 \%$ of the students could provide consistent answers in items $1 \mathrm{c}$ and $2 \mathrm{c}$.

With respect to the inconsistency observed in these items, $15 \%$ of the students provided correct explanations at the macroscopic level but misused the same principle at the microscopic level by stating that the number of accessible microstates remains unchanged. The number of students using the law correctly at the microscopic level and erroneously at the macroscopic level was lower, with only one student. These suggest that the macroscopic idea of the second law has been easier to grasp and this kind of inconsistency is observed as a result. The uncategorized and empty explanations in category 3 are meaningless to interpret as a result of the numerous possible explanations for those figures.

\section{Consistency of students' ideas concerning changes in entropy and the number of microstates for the Universe}

Students' ideas concerning changes in entropy or the number of accessible microstates for the Universe were tested at macroscopic and microscopic levels with items $1 \mathrm{~d}$ and $2 \mathrm{~d}$, respectively. The categorization of students' explanations for these items is shown in Table VII.

The categories seen in Table VII are typical of the ideas used by the students to explain these tasks, as introduced in Secs. III A 3 and III B 3. Descriptions of the categories are as follows:

TABLE VII. Cross tabulation of students' explanations concerning changes in entropy and in the number of accessible microstates for the Universe (items $1 \mathrm{~d}$ and 2d). $N=48$. Categories 1-5 are the same in columns and rows but for macroscopic (columns) and microscopic (rows) levels.

\begin{tabular}{lrrrrr}
\hline \hline & \multicolumn{5}{c}{ Macroscopic level } \\
\cline { 2 - 6 } Students' explanations & 1 & 2 & 3 & 4 & 5 \\
\hline \multicolumn{5}{c}{ Microscopic level } \\
1. Correct & $40 \%$ & $0 \%$ & $2 \%$ & $0 \%$ & $2 \%$ \\
2. Conserving quantity & $10 \%$ & $6 \%$ & $6 \%$ & $2 \%$ & $2 \%$ \\
3. The meaning of the & $2 \%$ & $0 \%$ & $4 \%$ & $0 \%$ & $0 \%$ \\
$\quad$ universe is not clear & & & & & \\
4. Size argument & $0 \%$ & $0 \%$ & $0 \%$ & $2 \%$ & $0 \%$ \\
5. Uncategorized and empty & $13 \%$ & $0 \%$ & $2 \%$ & $2 \%$ & $4 \%$ \\
\hline \hline
\end{tabular}


(1) Responses in this category correctly stated that the entropy of the Universe increases all the time.

(2) This category includes responses concluding that the entropy or the number of accessible microstates of the Universe would remain unchanged during the process.

(3) These responses indicate that students do not understand that the entities under inspection belong to the Universe.

(4) In these responses students stated that changes may be either impossible to determine or negligible due to the massive size of the Universe.

(5) These students gave either empty responses or responses of a type that could not be placed in these categories.

Questions regarding changes in the entropy and in the number of accessible microstates of the Universe were not trivial for the students: $40 \%$ of them were able to provide correct responses to the macroscopic and microscopic level items. $6 \%$ of the students utilized the idea of entropy as a conserved quantity consistently at both levels, which indicates that its nature is not understood correctly. $4 \%$ of the students misused the concept of the universe when providing explanations that revealed that they thought that the insulated systems under inspection did not belong to the Universe, consistently in both items. The size argument in category 4 was used consistently in both items by one student. These findings signal that $52 \%$ of the students were able to provide consistent answers in items $1 \mathrm{~d}$ and $2 \mathrm{~d}$.

Inconsistent responses were observed in several possible combinations of the categories. $10 \%$ of the students provided correct explanations for the items addressing change in the entropy of the Universe but they claimed that the number of accessible microstates of the Universe remained unchanged during the process. $6 \%$ of the students based their explanations at the macroscopic level on a misuse of the concept of the universe, and the same was the case with regard to their explanations at the microscopic level on an idea related to entropy conservation. These findings show that the conservation of the number of accessible microstates is a rather deeply rooted idea often used inconsistently with other ideas. Other evidently inconsistent ideas were used only by a few students, and hence these are omitted from this discussion.

One significant finding was that the percentage of uncategorized and empty explanations was very much larger at the microscopic level than at the macroscopic level, at $21 \%$ compared to $8 \% .13 \%$ of the students provided an acceptable response at the macroscopic level, but they could not provide an explanation at the microscopic level, which may indicate that the macroscopic level explanation has been easier to grasp for students than its microscopic counterpart.

\section{Summary}

Our most essential finding is that a majority of the students, between $52 \%$ and $69 \%$, depending on the item, used the second law of thermodynamics consistently at both the macroscopic and microscopic levels, either correctly $(38 \%-42 \%$, depending on the system under evaluation) or erroneously (12\%-27\%). However, some evident inconsistencies were also revealed. The most common inconsistency, harbored by $10 \%-15 \%$ of the students, was a tendency to consider that the number of accessible microstates remained constant even if they also stated that the entropy would increase. The opposite set of ideas, where the nature of the second law was understood correctly at the microscopic level but erroneously at the macroscopic level, was only witnessed in one pair of responses provided by one student. In addition, in the case of a few students, some inconsistencies related to the nature of increasing entropy and the meaning of the universe were also observed.

\section{DISCUSSION}

The second law of thermodynamics sounds relatively simple when written down: "Any large system in equilibrium will be found in the macrostate with the greatest entropy" [1]. Then again, a profound understanding of the law requires understanding both macroscopic- and microscopic-level explanations and the concepts of equilibrium, macrostate, and entropy. In this article, we have evaluated the consistency of students' ideas concerning the second law at both macroscopic and microscopic levels.

The significance of this study emerges partially from the universal importance of the physics content addressed. The actual meaning of the second law of thermodynamics and the concept of entropy can only be grasped with the aid of microscopic models and the concept of multiplicity. We claim that the meaning of the concept of energy cannot be fully understood without understanding the role of the second law in the degradation of energy. Hence, we would suggest that this type of universal principle is part of the most essential content for both scientists and teachers. Moreover, only with a consistent set of ideas can one be sure to explain physical phenomena correctly; we imagine that most scientists would agree with this statement.

Our findings concerning students' ideas about the second law and the nature of entropy agree well with previous studies, but there are also differences. The widely reported problem about referring to entropy as a disorder [3-8] was practically absent from our study: only one student used this kind of explanation. It is probable that modern textbooks [1] and teachers' familiarity with the previous studies have prevented this mistaken idea from dominating in students' thinking. Another typical mistaken idea of seeing entropy as a conserved quantity $[3,7,9]$ was also detected in this study. As in the case of earlier studies, some 
evidence exists that students confuse entropy and energy or other concepts, but simply to use the conservation argument is insufficient for causing that conclusion to be drawn [4,6-9]. The mistaken idea of thinking of entropy as something that will always increase locally was seen in our study, but only on the part of a few students $[3,6,9,10]$. With respect to understanding the meanings of other important concepts related to the second law of thermodynamics, such as system, surroundings, and the universe, some students gave explanations that suggested that they had confused surroundings and the universe [9].

Our research question, How consistent is students' use of the second law of thermodynamics at macroscopic and microscopic levels in the context of heat transfer processes?, has to be answered in a descriptive rather than a declaratory manner. When evaluating the consistency of students' responses to the items related to the individual entities, most students seem to have consistent ideas. It could also be seen that the principle of increasing entropy was sometimes applied erroneously and yet consistently, but there were also cases where it was used inconsistently. When evaluating students' use of the second law to a system consisting of two entities exchanging energy, a large majority of students responded consistently, in both correct and erroneous manners. However, plenty of the students possessed apparent inconsistencies at macroscopic and microscopic levels. This idea was confirmed when students' responses to the items addressing changes in the Universe were evaluated: in this case, a large percentage of the students' responses could be placed in various inconsistent combinations of categories.

These findings support the previous findings concerning the consistency of students' ideas, even if the percentages of inconsistent ideas are not as high as in some of the earlier studies [18]. On the other hand, straightforward comparisons are meaningless, since all of these studies have been in other fields of physics, using different approaches. The reason for the smaller degree of inconsistency remains unknown, but we think that it may be due to the similar contexts in our sets of items, or possibly because consistency was only evaluated by means of paired rather than multiple items. If the contexts were to be modified to render them further from each other, the result might be a different level of consistency. This offers an idea for another research project, where attention could be devoted to finding how similar contexts need to be for students' ideas to remain consistent.

With respect to the items, we admit that they may affect each other. Similar contexts can tempt students to consider the items in parallel, resulting in some impact on their responses. However, we claim that this should not be seen as a problem but rather as a demonstration of the existence of proficient skills in understanding and seeing connections. If a student sees the similarities and is able to provide correct responses on their basis, we should not see this as something that disturbs our ability to acquire a realistic view of students' ideas but rather as a valuable skill that may show that these students seek consistency.

Our results, like those of other studies [18-22], raise important questions about addressing different levels of explanation in teaching. For example, what is likely to offer a better starting point for learning: inconsistent but partially correct ideas or consistent, yet erroneous, ideas? It also causes us to consider the kind of strategies that can be used to reach consistency and correctness in terms of physics. Concerning the first of these questions, perhaps the correct part (e.g., a correct idea about the second law at macroscopic level) could work as an anchoring conception [32] that would help to transform the erroneous part (e.g., an erroneous idea about the second law at microscopic level) so that both consistency and correctness could be achieved. Then again, perhaps the consistent but erroneous ideas could be transformed to be correct with the aid of cognitiveconflict-based instruction, and the whole set of consistent ideas could then move "all at once."

Inconsistencies of this kind should be acknowledged by lecturers; it is not enough to state that students' content knowledge is correct in one context or at one level of evaluation (macro versus micro) but that their ideas should have explanatory power in various contexts, and in this particular case at different levels of evaluating a situation. In the following, we spell out some of the implications for teaching so that potential inconsistencies would not arise, or that they could be overcome. Despite the context of thermal physics, some of the following implications might also be useful in teaching other physics topics, especially those with a strong macro-micro connection, such as dc circuits [33].

We suggest that lecturers should utilize tasks that challenge students to evaluate phenomena at the macroscopic and microscopic levels concurrently. This would keep the connection between macroscopic and microscopic levels, i.e., entropy and the number of accessible microstates, in the forefront of attention and thus provide help in grasping the principle of the matter. Moreover, students should also be helped in detecting conflicts in their responses, possibly with tasks developed specifically for that purpose. This is one part of metaconceptual awareness that our students are expected to develop during their university studies [34]. We also think that findings from the field of physics education research (PER) concerning students' erroneous ideas may contribute to the design of tasks for this purpose; problematic themes may well provide contexts in which students could challenge their ideas and thus discover potential conflicts. However, designing tasks of this kind is far from straightforward, and hence it would probably be unreasonable to expect materials of this kind to be produced by individual lecturers. As a consequence, we suggest that textbook authors should place more emphasis on PER findings, as has already been done by a few authors [2]. 
We should emphasize the importance of evaluating entropy changes for individual entities, for combined systems consisting of multiple entities experiencing spontaneous changes, and for the Universe. Only when one is able to explain the changes in three different cases can one achieve a profound understanding of the second law. Special attention should be paid to the use of phrases familiar from textbooks, such as "entropy always increases," because they cannot provide adequate explanations in every single case. Hence, phrases of this kind should always be used with caution.

Based on our results, the classical macroscopic nature of entropy is understood better than the statistical nature of entropy that emerges from the microscopic approach. We claim that a thorough grasp of the microscopic interpretation of the second law may guarantee understanding of the macroscopic second law, which would then have significant implications for teaching. If a lecturer is successful in teaching the law microscopically, it is likely that students would be able to grasp the macroscopic version as well. Then again, our results suggest that grasping the law macroscopically does not seem to require a correct understanding of its microscopic counterpart. This might indicate that, for pragmatic purposes of the second law of thermodynamics, such as discovering the limitations that it poses for technological innovations, an understanding of the microscopic or statistical approach is not always required. Hence, we would suggest that for professions concentrating on applying the second law, such as engineering and chemistry, it might be a good idea to concentrate solely on the macroscopic interpretation and omit the microscopic approach entirely.

When the concept of entropy is constructed from a microscopic point of view, it relies heavily on analogies and abstract models of matter. Moreover, mathematical tools required at the microscopic level may be difficult to grasp due to their unfamiliarity. We claim that the profound problem lies in the transfer between these two levels: the connection between macroscopic- and microscopic-level explanations is difficult to grasp precisely because of the large step between the two levels. Moreover, this transfer requires assumptions and approximations that might confuse students even more. We suggest that lecturers should place a special emphasis on the problems involved while teaching this transfer. First, the most essential limitations concerning the analogies and models should be made clear to students when they are constructing the concept of entropy. Second, the transfer should not be undertaken too hastily but rather in smaller steps, so as to provide students with the time to grasp the "inevitability" of the law to increase together with the system size. This, in turn, can be related to the idea of thermodynamic limit, which we regard as an essential concept in the pursuit of a consistent and correct understanding of the second law.

\section{APPENDIX:}

The diagnostic test used to probe student learning. The desired responses expected from the students are written in italic style to separate them from the test questions. The first set of item questions has been adapted from Christensen et al. [9].

(1) An object is placed in a thermally insulated room that contains air. The object and the air in the room are initially at different temperatures. The object and the air in the room are allowed to exchange energy with each other, but the air in the room does not exchange energy with the rest of the world or with the insulating walls.

(a) During this process, does the entropy of the object $\left(\mathrm{S}_{\text {object }}\right)$ increase, decrease, remain the same, or is this not determinable with the given information? Explain your answer.

Desired response: This is not determinable because it depends on whether the object is at a higher or lower temperature than the air in the room. The entropy of the object increases if it receives energy and decreases if it gives out energy.

(b) During this process, does the entropy of the air in the room $\left(\mathrm{S}_{\text {air }}\right)$ increase, decrease, remain the same, or is this not determinable with the given information? Explain your answer.

Desired response: This is not determinable because it depends on whether the object is at a higher or lower temperature than the air in the room. The entropy of the air in the room increases if it receives energy and decreases if it gives out energy.

(c) During this process, does the entropy of the object plus the entropy of the air in the room $\left(\mathrm{S}_{\text {object }}+\mathrm{S}_{\mathrm{air}}\right)$ increase, decrease, remain the same, or is this not determinable with the given information? Explain your answer.

Desired response: It increases. Heat transfer is a spontaneous process, and the total entropy always increases in spontaneous processes; i.e., entropy of the sub-system receiving energy increases more than the entropy of the subsystem giving out energy decreases.

(d) During this process, does the entropy of the Universe $\left(\mathrm{S}_{\text {universe }}\right)$ increase, decrease, remain the same, or is this not determinable with the given information? Explain your answer.

Desired response: The Universe is an isolated system, and its entropy increases in all processes. Moreover, the object and the air in the room are part of the Universe so they also increase the entropy of the Universe.

(2) Two objects, $A$ and $B$, are placed in an insulated vessel, and the objects are connected with a thin 
metal bar (Fig. 1). Energy is transferred spontaneously from $A$ to $B$. What happens to the possible number of accessible microstates (increases, decreases, or remains unchanged) during the process in the following cases? Explain your responses, and refer to entropy whenever required.

(a) The number of accessible microstates of object $A$

Desired response: It will decrease because there are fewer possible ways for the energy quanta to distribute between the particles.

(b) The number of accessible microstates of object $B$ Desired response: It will increase because there are more possible ways for the energy quanta to distribute between the particles.

(c) The number of accessible microstates of the combined system of $A$ and $B$

Desired response: It will increase because the whole system of $A+B$ moves towards the most

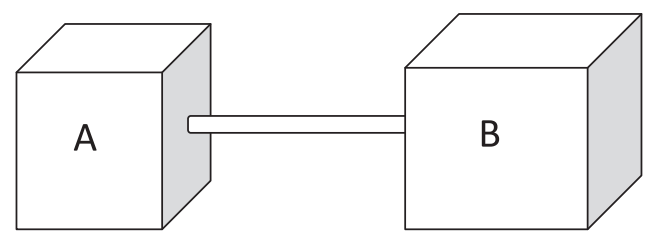

FIG. 1. Two objects connected with a thin metal bar.

probable state, i.e., the macrostate with most corresponding microstates.

(d) The number of accessible microstates of the Universe

Desired response: It will increase because, like the entropy, the number of microstates of the Universe always increases in all processes. The number of microstates is related to entropy in terms of the following equation: $S=k \ln \omega$, where $S=$ entropy, $k=$ Boltzmann constant, and $\omega=a$ number of microstates.
[1] D. V. Schroeder, An Introduction to Thermal Physics (Addison-Wesley, San Francisco, 2000).

[2] R. D. Knight, Physics for Scientists and Engineers-A Strategic Approach, 3rd ed. (Pearson, San Francisco, 2013).

[3] G. T.C. Ribeiro, Entropy and the second principle of thermodynamics-Fourth year undergraduates' ideas, Res. Assess. 9, 23 (1992).

[4] E. M. Carson and J. R. Watson, Undergraduate students' understandings of entropy and Gibbs free energy, U. Chem. Ed. 6, 4 (2002).

[5] D. F. Styer, Insight into entropy, Am. J. Phys. 68, 1090 (2000).

[6] B. R. Bucy, J. R. Thompson, and D. R. Mountcastle, What is entropy? Advanced undergraduate performance comparing ideal gas processes, in Proceedings of the Physics Education Research Conference, Salt Lake City, 2006 (American Association of Physics Teachers, American Institute of Physics, Melville, NY, 2006).

[7] M. Sözbilir and J. M. Bennett, A study of Turkish chemistry undergraduates' understandings of entropy, J. Chem. Educ. 84, 1204 (2007).

[8] A. H. Johnstone, J. J. MacDonald, and G. Webb, Misconceptions in school thermodynamics, Phys. Educ. 12, 248 (1977).

[9] W. M. Christensen, D. E. Meltzer, and C. A. Ogilvie, Student ideas regarding entropy and the second law of thermodynamics in an introductory physics course, Am. J. Phys. 77, 907 (2009).

[10] P. L. Thomas and R. W. Schwenz, College physical chemistry students' conceptions of equilibrium and fundamental thermodynamics, J. Res. Sci. Teach. 35, 1151 (1998).
[11] T. J. Greenbowe and D. E. Meltzer, Student learning of thermochemical concepts in the context of solution calorimetry, Int. J. Sci. Educ. 25, 779 (2003).

[12] S. Kesidou and R. Duit, Students' conceptions of the second law of thermodynamics-An interpretive study, J. Res. Sci. Teach. 30, 85 (1993).

[13] M. J. Cochran and P. R. L. Heron, Development and assessment of research-based tutorials on heat engines and the second law of thermodynamics, Am. J. Phys. 74, 734 (2006).

[14] C. H. Kautz, P. R. L. Heron, M. E. Loverude, and L. C. McDermott, Student understanding of the ideal gas law, part I: A macroscopic perspective, Am. J. Phys. 73, 1055 (2005).

[15] C. H. Kautz, P. R. L. Heron, P.S. Shaffer, and L. C. McDermott, Student understanding of the ideal gas law, part II: A microscopic perspective, Am. J. Phys. 73, 1064 (2005).

[16] K. Monteyne, B. L. Gonzalez, and M. E. Loverude, An interdisciplinary study of student ability to connect particulate and macroscopic representations of a gas, in Proceedings of the 2008 Physics Education Research Conference, Edmonton, 2008 (American Association of Physics Teachers, American Institute of Physics, Melville, NY, 2008).

[17] M. E. Loverude, Investigating student understanding for a statistical analysis of two thermally interacting solids, in Proceedings of the 2010 Physics Education Research Conference, Portland, 2010 (American Association of Physics Teachers, American Institute of Physics, Melville, NY, 2010).

[18] A. Tongchai, M. D. Sharma, I. D. Johnston, K. Arayathanitkul, and C. Soankwan, Consistency of 
students' conceptions of wave propagation: Findings from a conceptual survey in mechanical waves, Phys. Rev. ST Phys. Educ. Res. 7, 020101 (2011).

[19] M. Finegold and P. Gorsky, Students' concepts of force as applied to related physical systems: A search for consistency, Int. J. Sci. Educ. 13, 97 (1991).

[20] E. E. Clough and R. Driver, A study of consistency in the use of students' conceptual frameworks across different task contexts, Sci. Educ. 70, 473 (1986).

[21] D. Palmer, How consistently do students use their alternative conceptions?, Res. Sci. Educ. 23, 228 (1993).

[22] J. R. Watson, T. Prieto, and J. S. Dillon, Consistency of students' explanations about combustion, Sci. Educ. 81, 425 (1997).

[23] R. Gutierrez and J. Ogborn, A causal framework for analysing alternative conceptions, Int. J. Sci. Educ. 14, 201 (1992).

[24] J. Ogborn and J. Bliss, A psycho-logic of motion, Eur. J. Psychol. Educ. 5, 379 (1990).

[25] P. Nieminen, A. Savinainen, and J. Viiri, Relations between representational consistency, conceptual understanding of the force concept, and scientific reasoning, Phys. Rev. ST Phys. Educ. Res. 8, 010123 (2012).

[26] J. Leach, R. Millar, J. Ryder, and M.-G. Sére, Epistemological understanding in science learning: The consistency of representations across contexts, Learn. Instr. 10, 497 (2000).
[27] G. Driessen and P. Sleegers, Consistency of teaching approach and student achievement: An empirical test, School effectiveness and school improvement 11, 57 (2000).

[28] R. Leinonen, E. Räsänen, M. A. Asikainen, and P. E. Hirvonen, Students' pre-knowledge as a guideline in the teaching of introductory thermal physics at university, Eur. J. Phys. 30, 593 (2009).

[29] P. Mayring, Qualitative content analysis, Forum Qual. Soc. Res. 1, 20 (2000).

[30] S. Elo and H. Kyngäs, The qualitative content analysis process, J. Adv. Nurs. 62, 107 (2008).

[31] See Supplemental Material at http://link.aps.org/ supplemental/10.1103/PhysRevSTPER.11.020122 for authentic quotes of the explanations used by the students in various categories.

[32] J. Clement, Using bridging analogies and anchoring intuitions to deal with students' preconceptions in physics, J. Res. Sci. Teach. 30, 1241 (1993).

[33] R. W. Chabay and B. A. Sherwood, Matter \& Interactions, 3rd ed. (John Wiley \& Sons, New York, 2011).

[34] M. A. Asikainen and P. E. Hirvonen, A study of pre- and inservice physics teachers' understanding of photoelectric phenomenon as part of the development of a researchbased quantum physics course, Am. J. Phys. 77, 658 (2009). 